\title{
An Intelligent Medicine Bottle for Dropping Pills
}

\author{
Haihua Fu \\ Guangdong Mechanical and Electrical Polytechinc, Guangzhou, Guangdong Province, China
}

Keywords: Dropping pill, Intelligent, Medicine bottle, Structure, Principle

\begin{abstract}
This paper aims to study the reliable, safe and accurate medicine taking from bottles under normal conditions. At first, a sub-bottle was embedded on the cap of an ordinary medicine bottle. Then a control module was set between the sub bottle and the main bottle. Finally, the researcher marked the digital line and numbers from 0-10 on the outer edge of the cap evenly, and made a triangle mark below the digital line on the outer wall of the main bottle. When taking the medicine, the control module and metering device work together to control the quantity of medicine accurately. When the cap was rotated once, 10 drops could be obtained. The operation is simple, accurate, safe and healthy.
\end{abstract}

\section{Introduction}

At present, commonly used drug packages in the market include bag, bottle, aluminium plastic plate, tube, and so on.[1] No matter which form of packaging is adopted, most granular drugs (including dropping pills, tablets, capsules, and so on) are put into the mouth through contact, which brings patients the risk of secondary infection. At the same time, most of the packages lack sub safety and protection measures; children may take the medicine by mistakes. With China entering WTO, the state puts strict requirements on the drug industry, in order to nip in the bud the meet the needs of internationalized packaging. Under Jinping Xi's innovative theory, intelligent, environmentally friendly, safe, convenient, efficient, affordable and humanized packages are being explored and developed by WTO. For example, the Intelligent Container Equipment designed and developed by Jiandong Huang and Jianzhong Zhang of Wuxi Huifang Technology Co., Ltd. [2] and the Intelligent Medicine Box Having Automatically Dispensing and Monitoring and Reminding Functions[3] designed and developed by Jie Ji and Fanzhen Kong of Jining University have obtained the invention patent certificates.

\section{Design Objectives}

Pills have the features of regular shape and good rotary extrusion state [4]. In line with the spirit of promoting the traditional medicine culture of China, this design starts from the needs of patients at all levels, and adheres to the intelligent design concept of environmental protection, safety, convenience, high efficiency and affordable, and the humanized design principle of people-oriented, so as to ensure the basic functions of drug bottles in protection, convenient storage and transportation, promoting drug sales, and facilitating drug information identification.[5] An intelligent drug taking bottle for dropping pills is designed in this paper; it is accurate in taking medicine, healthy, safe and reliable.

\section{Basic Structure and Working Principle of the Intelligent Medicine Bottle for Dropping Pills}

\subsection{Basic Structure of the Intelligent Medicine Bottle for Dropping Pills}

The intelligent bottle is mainly composed of the control device, the metering device and the auxiliary device. The control device consists of fixed components and rotating components; the metering device is composed of a measuring mark and measuring numbers; the auxiliary device includes two parts: the main bottle and the sub bottle. 


\subsubsection{Control Device}

As shown in Figure 1, the fixed components are mainly composed of the inner bottle cap, the lower half of the middle bottle, the wheel and the control block slice.

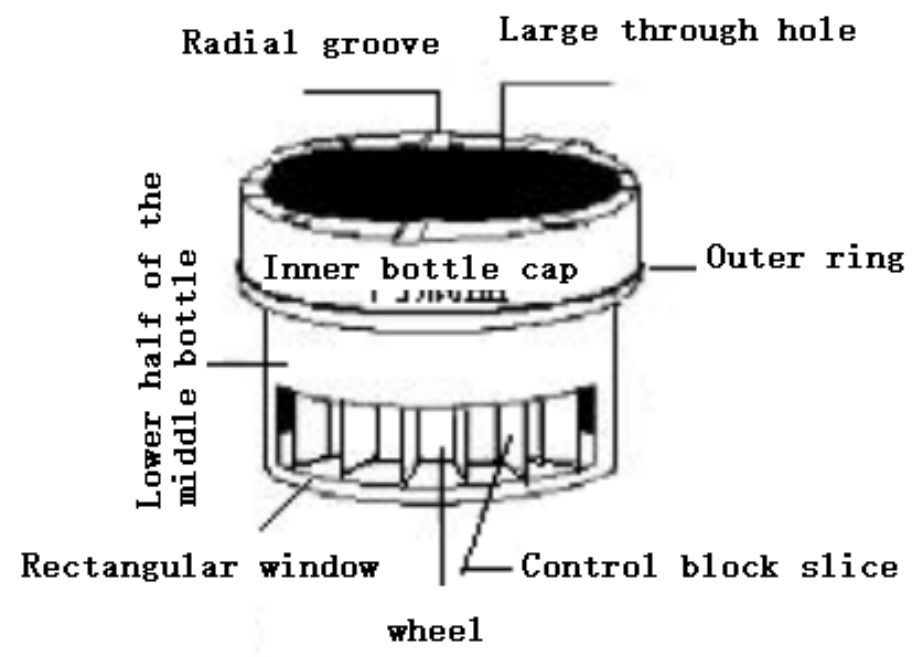

Fig.1 Fixed Components.

There is a large through hole in the middle of the inner bottle cap. After cutting off the upper part of the middle bottle, the middle bottle is fixed under the hole. A rectangular window (medicine window) is opened on the bottle wall near the bottom. A wheel is fixed at the centre of the bottom of the middle bottle. Within the specified angle range of the wheel circumference, six blocking slices are installed radially with an equal angle. Blocking slices are facing the rectangular window; their height is the same as the wheel; the width is slightly larger than that of the dropping pill by $0.5 \mathrm{~mm}$. A uniformly distributed radial groove is arranged on the upper end face of the ring of the bottle cap, which is used for tightening and screwing down the inner bottle cap when the radial shoulder corresponding to the annular lower end face of the outer bottle cap falls into the groove. The outer diameter of the middle bottle is slightly smaller than that of the main bottle (about 0.1 $\mathrm{mm}$ ). The inner bottle cap is fixed on the mouth of the main bottle through the internal thread, and there is a sealing washer between the two to ensure the reliable sealing of the main bottle.

As shown in Figure 2, the rotating component is mainly composed of the outer bottle cap and sub bottle.

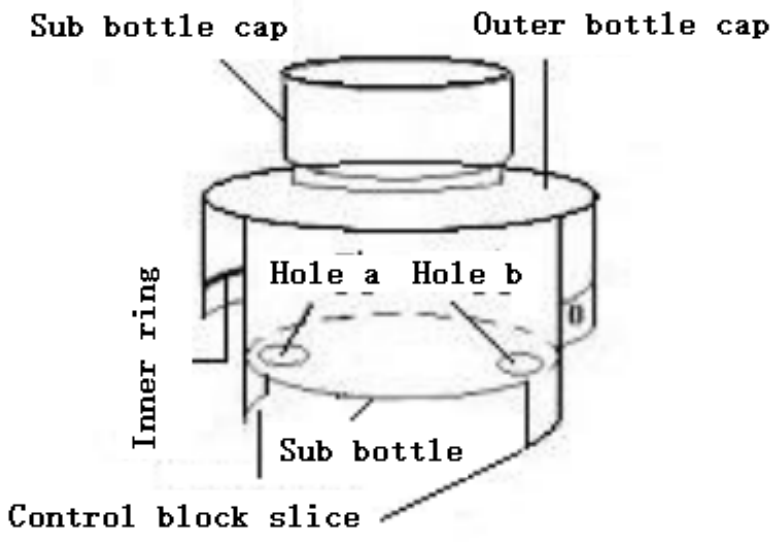

Fig.2 Rotating Components.

There is also a large through hole in the middle of the outer bottle cap, and the neck of the sub bottle is clamped in the hole. There are two small round holes (hole a and hole b) at the specified angle near the bottom at the wall of the sub bottle. The diameter of the hole is slightly larger than the diameter of the dropping pill. The bottle wall next to the small hole is provided with a control blocking slice which is longer than the diameter of a drop pill. The height from the lower end of the 
control block to the lower end face of the outer bottle cap is consistent with the height of the lower half of the middle bottle. The outer bottle cap is provided with an inner ring, which is used for axial positioning on the outer ring of the inner bottle cap during assembly. The outer diameter of the sub bottle and the inner diameter of the middle bottle are transitional fit, which plays the role of sealing and guiding.

\subsubsection{Metering Device}

As shown in Figure 3, Arabic numerals 0-10 and corresponding mark lines are marked on the circumference near the lower edge of the outer bottle cap, and a triangle mark is also marked at the bottle neck of the main bottle. They form the metering device of the intelligent medicine bottle for dropping pills.

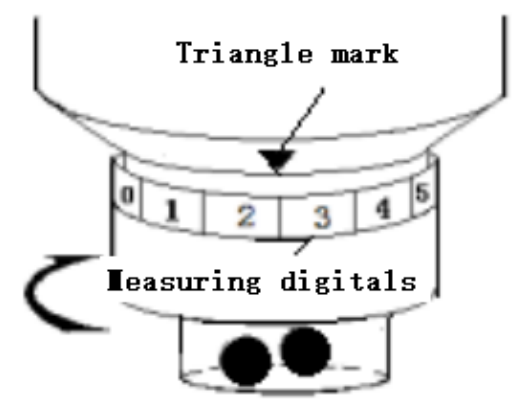

Fig.3 Metering Device.

\subsection{Working Principle of the Intelligent Medicine Bottle for Dropping Pills}

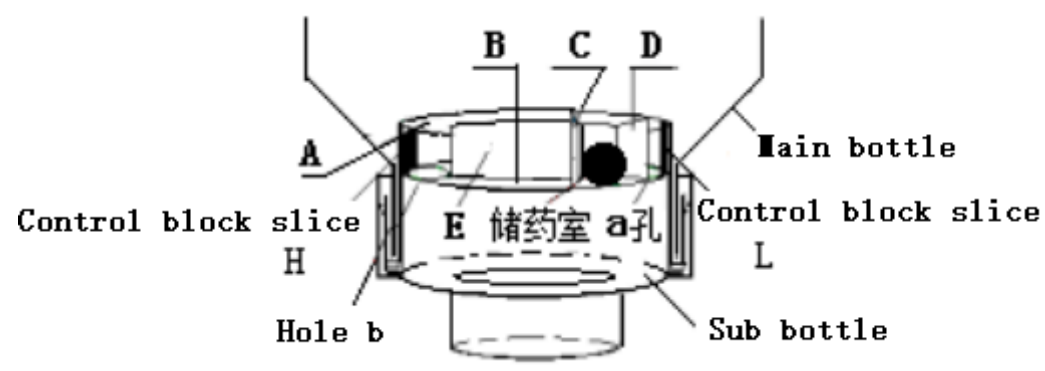

Fig.4 Drug Storage Chamber, Control Block Slice, Hole a and Hole B.

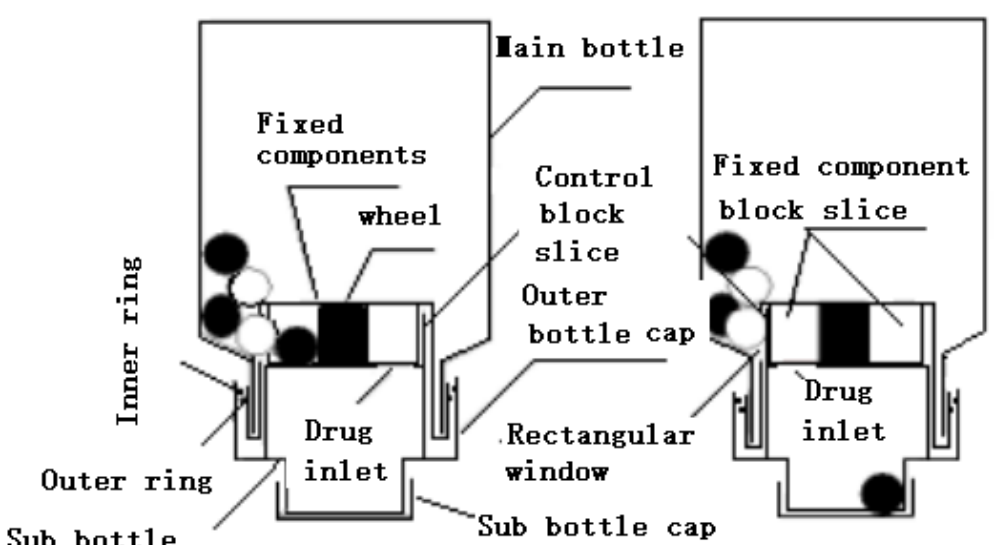

(a) Drug storage chamber

(b) Take medicine from hole a

Fig.5 The Working Principle of the Intelligent Bottle.

The process of drug taking is as follows. The patient screws the inner bottle cap of the fixed component of the control device on the mouth of the main bottle containing dropping pills, and then rotates and inserts the sub bottle with the outer bottle cap into the middle bottle of the fixed component until the inner ring of the outer bottle cap is clamped with the outer ring of the inner 
bottle cap (as shown in Figure 5 (a)). At that time, there should be a certain axial clearance between the inner and outer bottle caps, which can rotate relatively. When taking medicine, the patient first rotates the outer bottle cap until the " 0 " mark line on it is aligned with the triangle mark on the main bottle, then inverts the medicine bottle, pays attention to the measurement mark, and rotates the outer bottle cap clockwise until the number mark line corresponding to the drug quantity he needs is aligned with the triangle mark on the main bottle (as shown in Figure 3, the dosage is 2 pills). Finally, he rotates the sub bottle cap to open it, and pour pills in his mouth.

The working principle is as follows. As shown in Figure 4, when the inner bottle cap, the outer bottle cap and main bottle are closed, the outer bottle cap is rotated to the " 0 " mark line and aligned with the triangle mark on the main bottle. The open space formed by A surface of the bottom of the middle bottle, $\mathrm{B}$ surface of the bottom of the sub bottle, the $\mathrm{C}$ and $\mathrm{D}$ surfaces opposite to the two adjacent blocking slices installed on the wheel, and E surface of the outer cylindrical surface of the wheel, is called the drug storage chamber. There are five such drug storage chambers. The open side of the medicine storage chamber is connected with the inside of the main bottle through rectangular windows on the wall of the middle bottle (Figure 1 and Figure 5). Each storage chamber can only hold one dropping pill. When taking medicine, the main bottle is inverted; dropping pills in the main bottle naturally pass through the rectangular window along the bottle wall under its own gravity and enter into each medicine storage chamber (Figure 5 (a)). When the outer bottle cap is rotated clockwise, and the " 1 " mark line on the outer bottle cap is aligned with the triangle mark on the main bottle, the control block slice $\mathrm{L}$ on the wall of the sub bottle which is close to hole a will block the open side of the first drug storage chamber. At the same time, the small hole a on the B side at the bottom of the sub bottle just turns to the position of the first storage chamber. Therefore, the dropping pill in the first storage chamber will fall into the sub bottle through hole a. It is the first pill in the sub bottle. This process is called taking medicine, as shown in Figure 5 (b). If you continue to turn the outer bottle cap until the "2" mark on it is aligned with the triangle mark on the main bottle (Figure 3), in the same way, the space between the second medicine storage chamber and the main bottle space is separated by the control block slice L close to hole a, and the small hole a on the B surface at the bottom of the sub bottle is transferred to the position of the second medicine storage chamber. The second drop pill in the second storage chamber falls into the sub bottle through hole a, and there is the second drop pill in the sub bottle. At the same time, the first medicine storage chamber is opened because the control slice is transferred to the second medicine storage chamber; dropping pills in the main bottle enter the first medicine storage chamber along the bottle wall again. If the outer bottle cap is further rotated, the third medicine storage chamber takes medicine, the second medicine storage chamber stores medicine, and so on. When hole a finished taking the fifth dropping pill, the four drug storage chambers are filled dropping pills. At this time, hole $\mathrm{b}$ is rotated to the front of the first medicine storage chamber to prepare for taking medicine. When hole $b$ finished taking the pill for the first time, there were 6 pills in the bottle. Each time the outer bottle cap rotates, 10 pills can be taken from hole a and hole $b$ in total. We can repeat the cycle until the number of pills required by the patient is satisfied. Figure 6 is the intelligent bottle designed in this paper. The application practice has proved that the usage is quite smooth.

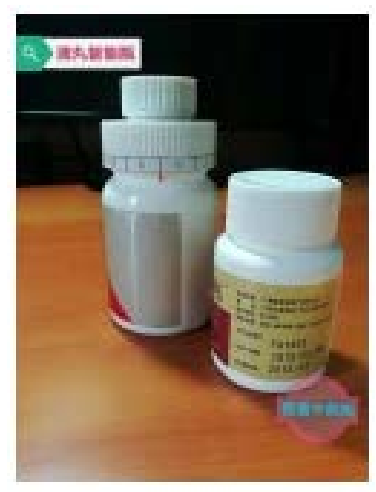

Fig.6 Intelligent Medicine Bottle for Dropping Pills. 
Due to its safe and simple operation, as well as accurate and healthy drug taking, the intelligent bottle has been granted a utility model patent by the National Intellectual Property Administration (Patent No.: ZL201721729855.3). The applied invention patent is currently in the process of actual trial and publicity (Patent No.: 2017113108111). The invention and innovation points are as follows.

It is safe for children. The package with barrier structure is designed by using the intelligent barrier class (method), and the drug taking is controlled by multiple mechanisms and specific actions, which can effectively reduce safety accidents of specific objects (children) eating and using dangerous goods by mistake. [6]

It is intellectualized and convenient. The intelligent bottle cap with metering function can help patients take drugs more conveniently. [7]

It is highly efficient. The bottle realizes accurate measurement through the metering device and the one-time drug delivery through the sub bottle or the sub bottle cap, with high medication efficiency and convenient drug access. [8] It has realized a breakthrough in the real and practical sense of measuring and taking drugs.

It is healthy. The scientific drug taking method can overcome patients' bad habit of taking medicine by hand, and effectively avoid the secondary pollution of drugs and infection of other diseases, so as to effectively protect the health and life safety of consumers. [9]

It is economic. The purely mechanical design is easy to manufacture and promote. The cost is low (0.3-0.8 yuan / piece, while the crowd funding price of iRemember smart medicine bottle on Indiegogo is only 35 US dollars (about RMB 215 yuan)), which fully conforms to the value and cultural orientation of consumers. [10]

The bottle has good sealing stability.[11] There is a sealing gasket between the inner bottle cap and the bottle body. When opening the sub bottle cap to take medicine, the air does not directly contact medicine in the main bottle; the oxidation and deterioration drug are blocked, and the stability can be guaranteed.

It has a wide range of application. The dosage can be accurately controlled, and it is suitable for patients of all ages. It also follows the people-oriented design concept in ergonomics, which makes things adapt to people rather than asking people to adapt to things. [12]

It is expandable. If the cost is allowed, a micro electronic reminder can be designed and installed on the bottle cap as the timing device, which can help the elderly take medicine on time and improve their compliance.[13]

\section{Conclusion}

At present, COVID-19, the novel coronavirus which causes fierce pneumonia, has swept the globe and began to devour the lives of our compatriots [14-15]. This reminds us of the frequent occurrence of influenza A (H1N1), Ebola in West Africa and "Zika" epidemic in recent years, which have seriously threatened human survival and development [16-18]. If human beings do not have scientific and effective measures to prevent the epidemic, the consequences will be unimaginable. The cruel reality tells people that food safety, public health and drug safety are not only related to our physical and mental health, but also related to the development and survival of human beings. Therefore, if the humanized and intelligent bottle for dropping pills is widely used, it can "solve the problems of unsanitary and single function in existing technology."[19] Moreover, it can solve world problems, such as preventing drug accidents among children caused by packaging problems, and certainly make due contributions to improving people's health and safety awareness, to revitalizing the traditional medicine culture of China and to promoting the development of human civilization.

\section{References}

[1] Hu, F.M. The Fact and the Future of The Medicine Package Development. China Packaging Industry, vol. 12, no. 26, pp.41-44, 2002. 
[2] Huang, J.D., Zhang, J.Z. Intelligent Container Equipment. China, 201410443817.6, 2016-08-24.

[3] Ji. J, Kong, F.Z., Wang, Q., et. al. Intelligent Medicine Box Having Automatically Dispensing and Monitoring and Reminding Functions. China, 201610058195.4, 2018-03-13.

[4] Guo, J., Zhang, J., Zhang, P.P., et. al. Pills Medicines Packaging Structure Design of Multifunctional Sealed Cover. Packaging Engineering, vol. 33, no. 11, pp. 88-90, 2012.

[5] Zhu, H.P., Cai, J.S., Ke, S.H. Research on Intelligent Control Drug Packaging Design. Hunan Packaging, vol. 32, no. 3, pp. 31-33, 2017.

[6] Wang, Y.Z., Ke, S.H. Packaging Design of the Obstacle Structure. Packaging Engineering, vol. 40, no. 9, pp. 107-112, 2019.

[7] Yu, X.Y. Huang, S.Y. The Intelligent Future of Pharmaceutical Packaging. Printing Technology, no. 22, pp. 22-23, 2014.

[8] Wu, X.L. Humanized Older Drugs Packaging Design Construction of Evaluation System. Packaging Engineering, no. 18, pp. 90-94, 2019.

[9] Liu, W.L., Hu, S.Y. Review on the Study of Safety Design in Pharmaceutical Packaging. Hunan Packaging, no. 6, pp. 11-15, 2018.

[10] Zhu, H.P., Yao, J. Research of the Intelligent Packaging Design: Take the Elderly Intelligent Drug Packaging as an Example. Art \& Design, no. 5, pp. 96-97, 2013.

[11] Zhu, Y. Environmental Protection Packaging for Building Safety Defence Science. Plastic Packaging, no. 04, pp. 10-16, 2019.

[12] Yu, Q. Humanized Design of Drug Packaging -- to Solve the Problem of Taking Medicine for the Elderly. China Packaging Industry, no. 16, pp. 29 - 31, 2013.

[13] Liang, C.W. Drug Safety Intelligent Packaging Design Strategy. Urban Construction Theory Research (The Electronic), no. 34, pp. 198, 2018.

[14] Severe Acute Respiratory Syndrome-Related Coronavirus-the Species and its Viruses, a Statement of the Coronavirus Study Group, [online] Available: https://www.biorxiv.org/content/10.1101/2020.02.07.937862v1.

[15] National Health Committee about the Revised New Coronavirus Pneumonia English Name of the $\quad$ Notice, [online] Available: http://www.nhc.gov.cn/xcs/zhengcwj/202002/6ed7614bc35244cab117d5a03c2b4861.shtml.

[16] Wang, Y.H., Deng, M. Advances in the Etiology and Pathogenesis of Influenza A (H1N1). Chinese Journal of Nosocomiology, vol. 20, no. 24, pp. 4047-4048, 2010.

[17] Chu, Y.Z. Progress Toward Poliomyelitis Eradication-Nigeria, January 2013-September 2014. Chinese Journal of Vaccines and Immunization, vol. 21, no. 04, pp. 471-472, 2015.

[18] Huang, C., Ma, H.X., Liang, H.G., et. al. Global Countermeasures Against Ebola Virus Disease and Enlightenment on Preventing and Controlling Fulminating Infectious Diseases in China. Military Medical Sciences, vol. 42, no. 10, pp. 786-791, 2018.

[19] Yang, Y., Pang, C.Y., Huang, Z.L., et. al. Automatic Medicine Discharging and Automatic Recording Intelligent Medicine Bottle. China, 201610436058.X, 2019-01-22. 